\title{
Obesity surgery and eating and weight disorders: a new topical collection of EWD
}

\author{
Gianfranco Silecchia ${ }^{1}$
}

Received: 19 May 2020 / Accepted: 19 May 2020 / Published online: 26 May 2020

(c) Springer Nature Switzerland AG 2020

Keywords Obesity surgery $\cdot$ Metabolic surgery $\cdot$ Eating and weight disorders

It is a pleasure and honor to accept the invitation of the Editor-in-chief to introduce and to launch the new topical collection on obesity surgery and eating and weight disorders.

Bariatric/metabolic surgery represents the most effective therapeutic modality for morbid obesity and related major co-morbidities with long-term results, including sustained weight loss and co-morbidities control/remission. Metabolic surgery achieves reduction of the mortality rate $(45 \%)$, cardiovascular events (65\%) and DMT2 (60\%) [1-5].

However, the way out from morbid obesity (chronic disease) also for the bariatric population is not easy. Three steps are crucial for favorable long-term outcomes:

1. patients selection (multidisciplinary team involvement mandatory)

2. intensive peri-operative period monitoring (1-3 months)

3. end of bariatric surgery "honeymoon" (1-2 years) and risk of follow-up dropout and weight regain.

There is a worldwide agreement on the essential role of the multidisciplinary team (obesiologist, endocrinologist, cardiologist, pneumologist, psychologist, registered dietician and bariatric surgeon) in the weight loss program. Moreover, in a bariatric surgical center, it is mandatory the "permanent" support by a nutritionist/registered dietician and a psychologist or, when appropriate, a psychiatrist. The multidisciplinary management is advocated to guarantee the best long-term outcomes after all the different bariatric procedures.

Gianfranco Silecchia

Gianfranco.silecchia@uniroma1.it

1 Department of Medico-Surgical Sciences and Biotechnologies, Faculty of Pharmacy and Medicine, Sapienza University of Rome, Corso della repubblica 79, 040100 Latina, Italy
The perioperative period is a "critical zone" and the psychological events are often under-evaluated: reactions to the loss of food, regrets having surgery, relationship changes, fear of weight regain, addiction transfer such as alcohol abuse. After that, several studies reported maladaptive eating behaviors after bariatric surgery that may contribute to weight regain.

The diagnosis and management of new-onset disordered eating behaviors after metabolic/bariatric surgery represent a new field of multidisciplinary research. There is a highlight on the increasing evidence of new eating and psychological disorders in the subset of bariatric patients. The bariatric surgery represents a new model for the gastrointestinal physiology and a potential pandora box of eating patterns and disorders.

Insufficient weight loss and weight regain are reported by up to $25-30 \%$ after bariatric surgery [4]. Guidelines for multidisciplinary management and robust evidence on management are lacking and offer an opportunity for future research.

The patients require an "alliance" among the professionals involved in the "three crucial steps". Evidence advocates strong cooperation between dietician/nutritionist and mental health professionals to fight against morbid obesity as a chronic disease. Relapse also after surgery is a hot topic, and validated management protocols are needed.

The topical collection aims to provide an overview of the latest research findings on bariatric/metabolic surgery and eating patterns/disorders, insufficient weight loss, and weight regain after surgery. Contributions related to the following non-exhaustive areas are encouraged:

1. revisional bariatric/metabolic surgery for weight regain and/or insufficient weight loss

2. eating patterns/disorders, addiction transfer such as alcohol abuse after bariatric surgery

3. multidisciplinary team in bariatric surgery program 
4. long-term surgical complications after bariatric surgery and emerging maladaptive eating disorders

5. taste and eating habits after sleeve gastrectomy, standard gastric bypass, and one anastomosis gastric bypass

6. emerging hypo-absorptive procedure and need of supplementation

7. news endoscopic bariatric procedures and multidisciplinary management

8. obesity surgery and obesity management during Covid19 pandemic.

EWD should be the "target" international journal for research on bariatric populations focused on eating patterns and disorders after bariatric surgery. The surgeons should understand the significant role of psychological events after surgery and pay great attention to the new-onset of unexpected eating behaviors involving mental health professions in the postoperative management. Psychologists and nutritionist/dieticians should consider bariatric population as a challenging cohort and adopt a new evidence-based approach.

I hope that the new topical collection will stimulate investigators to submit original papers on obesity surgery and eating and weight disorders to EWD, a scientific journal born to be the "ideal home" for the multidisciplinary team approach in obesity treatment.

Funding None.

\section{Compliance with ethical standards}

Conflict of interest The author declares that he has no conflict of interest.

Ethical approval This article does not contain any studies with humans or animals performed by the authors.
Informed consent For this type of study formal consent is not required.

\section{References}

1. Sheng B, Truong K, Spitler H, Zhang L, Tong X, Chen L (2017) The long-term effects of bariatric surgery on type 2 diabetes remission, microvascular and macrovascular complications, and mortality: a systematic review and meta-analysis. Obes Surg 27:2724-2732. https://doi.org/10.1007/s11695-017-2866-4

2. Buchwald H, Buchwald JN (2019) Metabolic (bariatric and nonbariatric) surgery for type 2 diabetes: a personal perspective review. Diabetes Care 42:331-340. https://doi.org/10.2337/ dc17-2654

3. YanID G, Wang J, Zhang J, Gao K, Zhao Q, Xueqin X (2019) Long-term outcomes of macrovascular diseases and metabolic indicators of bariatric surgery for severe obesity type 2 diabetes patients with a meta-analysis. PLoS ONE 14(12):e0224828. https ://doi.org/10.1371/journal.pone.0224828

4. Di Lorenzo N, Antoniou SA, Batterham RL, Busetto L, Godoroja D, Iossa A, Carrano FM, Agresta F, Alarçon I, Azran C, Bouvy N, Ponz CB, Buza M, Copaescu C, De Luca M, Dicker D, Di Vincenz A, Felsenreich DM, Francis NK, Fried M, Prats BG, Goitein D, Halford JCG, Herlesova J, Kalogridaki M, Ket H, MoralesConde S, Piatto G, Prager G, Pruijssers S, Pucci A, Rayman S, Romano E, Sanchez-Cordero S, Vilallonga R, Silecchia G (2020) Clinical practice guidelines of the European Association for Endoscopic Surgery (EAES) on bariatric surgery: update 2020 endorsed by IFSO-EC, EASO and ESPCOP. Surg Endosc. https:// doi.org/10.1007/s00464-020-07555-y (online first 23/04/2020)

5. Capoccia D, Guida A, Coccia F, Guarisco G, Testa M, Leonetti F, Silecchia G (2020) Weight regain and diabetes evolution after sleeve gastrectomy: a cohort study with over 5 years of follow-up. Obes Surg 30:1046-1051. https://doi.org/10.1007/s11695-01904350-0

Publisher's Note Springer Nature remains neutral with regard to jurisdictional claims in published maps and institutional affiliations. 